\section{Mark A. Smith: Scholar and Innovator}

How could so much science, innovation, mentoring and service be concentrated in a life of 45 years and faculty career of less than 20 ? In the 1990s, Mark A. Smith entered neuroscience and shook the foundations of Alzheimer research, with the first compelling demonstration of oxidative damage and response in Alzheimer disease (AD). In December 2010, just as suddenly and more unexpectedly, he left the world, leaving behind a field in need.

While we will never know the divine plan that took him from us, we do know the rise. On entering the area, Mark realized that diffuse changes of oxidation dependent epitopes, insolubility, and qualitative analysis of heterogeneous tissue samples were hindering the field by obscuring the cellular complexity of $\mathrm{AD}$, so he set out to develop a comprehensive array of histochemical and antibody probes to identify in exquisite detail and specificity oxidative damage and response at the subcellular level. Having used these probes to define oxidative damage to every molecular class of biological molecule and essentially every response, Mark then set out to define the mechanism for oxidative changes that predate the pathophysiology. His efforts brought new concepts to the field: unscheduled cell cycle activation, the two-hit hypothesis, phosphorylation control of oxidative modification, the role of gonadotropins in neurodegeneration, cell death avoidance, oxidative stress response induction, and finally the concept that enveloped his work, that the pathology of $\mathrm{AD}$ is a protective response.

Mark was passionate in getting to the truth of the matter, fearlessly voicing his views to friends, supporting colleagues, mere acquaintances, and opposing authority figures alike, and was equally tireless in his efforts to disprove his own hypotheses. With all this wrapped into a single person, it is hard to imagine that he was the most prolific author in $\mathrm{AD}$ research and the fifth most cited. Or that he was head of the faculty union at Case (AAUP). Or that several highly prestigious teaching and mentoring awards joined his many research recognitions and accolades.

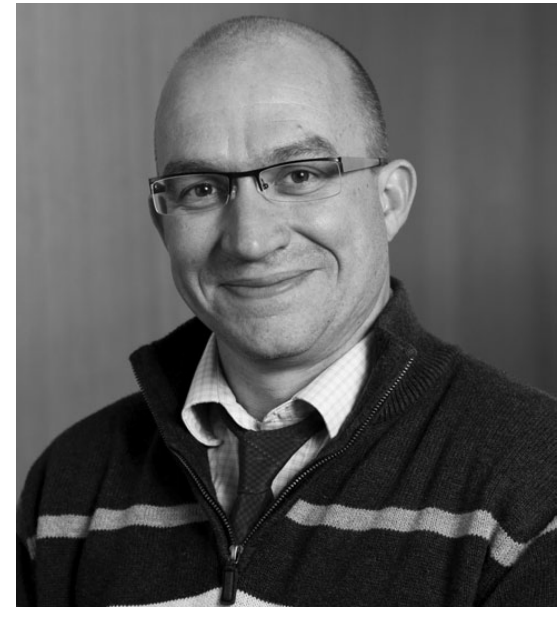

And with his death, those close to Mark mourn the loss, while the field will miss one if its greatest innovators.

Rudy Castellani, George Perry, Hyoung-gon Lee, Xiongwei Zhu 